\title{
QUANTIFICATION OF MICROSTRUCTURAL EVOLUTION AND TEXTURE DEVELOPMENT DURING RECRYSTALLIZATION
}

\author{
R. A. VANDERMEER ${ }^{1}$ and D. JUUL JENSEN ${ }^{2}$ \\ ${ }^{1}$ Code 6320.1, Naval Research laboratory, Washington D. C. 20375-5343, USA \\ ${ }^{2}$ Materials Department, Ris $\phi$ National Laboratory, DK-4000, Roskilde, Denmark
}

(Received 20 November 1995)

\begin{abstract}
During primary recrystallization a population of new grains nucleates and grows within a cold-deformed material until the latter is consumed. This process was studied from a combined microstructure, kinetics and texture viewpoint in commercial aluminum. Experimentally, recrystallization microstructures were quantified by stereological variables and Cahn-Hagel growth rates. The orientations of the grains being measured stereologically were identified and experimentally determined using the back-scatteredelectron pattern analysis. A geometrically-based, statistical mathematical model of recrystallization kinetics, formulated analytically on nucleation and growth premises, was devised taking into account both the behavior of individual recrystallization texture components and the complex geometrical impingement patterns due to recrystallized grains occurring in clusters. By correlating and matching the experimental stereological measurements with the analytical model, the nucleation behavior and growth rates during recrystallization were estimated for each texture component. A 3-D computer simulation verified the analytically-deduced microstructural and textural description of recrystallization and allowed the as-recrystallized grain sizes and the grain size distributions to be modelled as well. Using the combined approach, a complete nucleation and growth model for recrystallization of heavily cold rolled AA1050 was established.
\end{abstract}

KEY WORDS: Recrystallization, nucleation, growth, texture, microstructure, modelling, EBSP.

\section{INTRODUCTION}

Primary recrystallization of cold worked metallic materials is a complex, thermallyactivated process of microstructural evolution that depends upon many factors such as the composition of the material, the prior microstructure and texture, the nature and amount of deformation, the temperature of deformation, the time and temperature of annealing etc. Kinetically, the recrystallization process can be characterized in terms of two component phenomena - nucleation and growth. During nucleation, a population of new strain-free grains becomes distinguishable from the features of the deformation microstructure. As time proceeds, these new grains enlarge by means of grain boundary migration until the deformed volume is consumed and the excess stored energy due to cold work is removed. In heavily cold worked materials, substantial texture changes can accompany recrystallization, altering the properties of the material ( $\mathrm{Hu}, 1981)$.

Efforts to quantify the recrystallization process experimentally have typically followed one or the other of two paths. On the one hand, there have been numerous investigations of the kinetics of microstructural evolution during recrystallization by stereology (see for example Vandermeer and Rath $(1989 a, b)$ ) or by following changes 
in various physical properties (see for example Woldt and Juul Jensen (1995)). Also in the microstructural mode, others have concentrated on measuring the grain size after recrystallization is complete. On the other hand, much work has also been expended in describing the development of texture during recrystallization from x-ray or neutron diffraction experiments and analysis (see for example Beck and Hu (1966)) and the Proceedings of various ICOTOM Conferences).

Very recently, because of the advent of local orientation measurements in the electron microscope, it has become possible to combine these two separate paths into one and quantify recrystallization by characterizing the nucleation and growth behavior of the various texture components individually as well as in total (Vandermeer and Juul Jensen, 1994a, 1995). The purpose of this paper is to demonstrate an approach that may be utilized to address this possibility.

Essentially, the methodology consists of combining two aspects of investigation experimental observations and modelling. The two aspects are as follows: first, microstructural evolution during recrystallization is experimentally characterized, employing quantitative stereological analysis on a series of partially recrystallized specimens. The stereological properties of the microstructure that are measured are, $\mathrm{V}_{\mathrm{v}}$, the volume fraction recrystallized, $S_{\mathrm{v}}$, the interfacial area density separating recrystallized grains from deformed regions and $\langle\lambda\rangle$, the mean recrystallized grain chord length. At the same time the stereological data are being gathered by point and lineal counting, the orientations of the specific recrystallizing grains being counted are also determined by electron-back-scatter pattern (EBSP) analysis. All of this may be accomplished in a scanning electron microscope that is equipped with a traversing stage and is operated in the electron back scatter mode with an approximately $1 \mu \mathrm{m}$ beam spot. The collected orientation and stereological data are sorted and categorized according to the texture component to which they belong.

Second, geometrically-formulated, microstructural models premised on various nucleation and growth concepts are developed for each texture component being considered. The models are expressed analytically in terms of a set of nucleation and growth parameters and the same microstructural properties as those determined experimentally. The modelling and experimental aspects of investigation are combined by attempting to fit the experimental data to a nucleation and growth model. A model is not considered successful until a strict match is achieved. If no previously formulated model is found to be adequate, it may be necessary to develop a new one. Once the values of the parameters required to accomplish the matching of model and experiment are determined, they are used to assess the nucleation and growth rate behavior for each texture component. This method has been used to investigate the recrystallization of heavily rolled commercial aluminum (Vandermeer and Juul Jensen, 1994a) and copper (Vandermeer and Juul Jensen, 1995). The recrystallization modeling approach (including texture) that is employed here is microstructure based and differs from the approaches of others who use the Monte Carlo technique (Tavernier and Szpunar, 1991) or a mathematical analysis of orientation distribution functions with either an oriented nucleation or oriented growth hypothesis (Köhler and Bunge, 1994).

Up to now this two pronged approach to quantify nucleation and growth during recrystallization has concentrated mainly on characterizing the $V_{v}, S_{v}$ and their dependencies on time and on each other for the various texture components being considered. It has not yet been possible to deduce the mean recrystallized grain sizes from the nucleation and growth characteristics of the analytical models. In this paper we will show how to improve upon this methodology by introducing a third prong 
to the investigatory approach, namely the tool of computer simulation. We will seek to demonstrate how, with the addition of the computer simulation tool, a fuller textural and microstructural description (including the recrystallized grain size and size distributions) of recrystallization of a rolled, commercial AA1050 aluminum may be achieved leading to a more complete nucleation and growth description of the recrystallization process. A preliminary account (Juul Jensen and Vandermeer, 1995) of the application of this expanded method using the earlier aluminum data (Vandermeer and Juul Jensen, 1994a) has already been published. The aluminum used in this study, while similar in composition to the earlier study, was processed differently and annealed at a slightly higher annealing temperature.

\section{EXPERIMENTAL}

A commercial aluminum alloy AA1050 containing $0.32 \mathrm{wt} \% \mathrm{Fe}$ and $0.15 \mathrm{wt} \% \mathrm{Si}$ was continuously cast, homogenized at $600^{\circ} \mathrm{C}$ and slow cooled to $400^{\circ} \mathrm{C}$. After breakdown rolling and annealing, the alloy exhibited a $100 \mu \mathrm{m}$ grain size, a weak texture and a relatively uniform distribution of $1.7 \mu \mathrm{m}$ diameter particles comprising $0.5 \%$ of the volume. After a final deformation by rolling at room temperature to a reduction in thickness of $90 \%$ specimens for the recrystallization study were cut and annealed for times ranging from $120 \mathrm{~s}$ to $7200 \mathrm{~s}$ in a molten tin bath held at $280^{\circ} \mathrm{C}$. The penultimate microstructure of this aluminum alloy differed from the earlier one principally in the nature and distribution of the particles.

The partially recrystallized specimens were cut to expose a longitudinal transverse section which was polished mechanically, chemically and electrolytically for microstructural examination in a JEOL 840 scanning electron microscope. Linear traverses for stereological measurements were scanned with the electron beam in the rolling direction and the normal direction equally until at least 130 recrystallized grains were measured. From point and line counting during the traverse and EBSP measurements of the orientations of the grains encountered, values of the microstructural properties, $\mathrm{V}_{\mathrm{v}}(\mathrm{i}), \mathrm{S}_{\mathrm{v}}(\mathrm{i})$ and $\langle\lambda\rangle$ (i), where (i) refers to a specific texture component, were estimated. The recrystallized grains were categorized into one of three orientation classes. Cube grains were those whose observed orientations fell within 15 degrees of the cube orientation, i.e. $\{100\}<001\rangle$, while rolling grains were those with orientations within 15 degrees of the rolling texture components, i.e. $\{110\}<112>$, $\{123\}<634>$ or $\{112\}<111>$. The remaining recrystallized grains comprised the random orientation component. For additional experimental details about stereology and the EBSP determinations see Vandermeer and Juul Jensen (1994a).

\section{COMPUTER SIMULATION}

The geometric computer simulation method is a modification of the one first introduced by Mahin, Hanson and Morris (1980) and extended to include multiple texture components by Juul Jensen (1992). Essentially, a three dimensional "deformed specimen" is simulated by a cube containing a network of grid points. Within the cube, recrystallized grain nucleation sites are prescribed and nuclei added, either all at once at zero time or stepwise in time at a predetermined rate. A growth law is assigned to each nucleus. After a certain time step, each grid point is interrogated as 
to whether any recrystallized grain has reached it. The first grain to reach a grid point is assigned to it. After each step in a series of time steps, the interrogation and assignment process is repeated limited, however, to the grid points associated with a "plane of polish" passing through the cube thereby simulating a metallographic specimen. Periodic boundary conditions are maintained at the faces of the cube. The algorithm analyses the state of the gridpoints, "sketches" the microstructure, calculates both of the microstructural properties, $V_{v}$, and $S_{v}$ for each texture component and computes the mean recrystallized grain size and the grain size distribution for each component. Care is taken to ensure that the recrystallized grain size is small compared to the cube (specimen) size but large compared to the grid size when recrystallization is complete. Normally the "experiment" is repeated 5 times using the same time intervals but with different starting seeds for the distribution of nuclei. More details of the simulation procedure are given by Juul Jensen (1992).

\section{ANALYSIS}

The partially recrystallized microstructural data obtained from the deformed AA1050 aluminum and for which a recrystallization model based on nucleation and growth is sought, are tabulated in Table 1. There was little difference between the data gathered from the two scanning directions that were $90^{\circ}$ apart from each other so only a single weighted mean value (Hilliard (1966), Hilliard and Cahn (1961) and Beers (1957)) is listed for each microstructural property.

\section{Cahn-Hagel Growth Rates}

Spatially-averaged interface migration rates for each texture component were estimated from the Cahn-Hagel relationship (Cahn and Hagel, 1960, 1963)

$$
\langle v\rangle=\frac{1}{S_{v}} \cdot \frac{d V_{v}}{d t}
$$

Table 1 Microstructural Property Data for Recrystallization of AA1050 Aluminum.

\begin{tabular}{llllllr}
\hline Property* & \multicolumn{7}{c}{ Annealing Time (sec) } \\
\cline { 2 - 7 } & 120 & 300 & 600 & 1800 & 3600 & 7200 \\
\hline & 0.0123 & 0.0357 & 0.0674 & 0.1798 & 0.4669 & 0.5829 \\
$\mathrm{~V}_{\mathrm{v}}$ random & 0.0133 & 0.0301 & 0.0278 & 0.0688 & 0.0467 & 0.0066 \\
$\mathrm{~S}_{\mathrm{v}}$ random & 2.723 & 3.083 & 4.329 & 5.808 & 11.888 & 11.273 \\
$<\lambda>$ random & 0.0043 & 0.0142 & 0.0318 & 0.0992 & 0.1710 & 0.2543 \\
$\mathrm{~V}_{\mathrm{v}}$ rolling & 0.0044 & 0.0105 & 0.0171 & 0.0323 & 0.0195 & 0.0025 \\
$\mathrm{~S}_{\mathrm{v}}$ rolling & 2.342 & 2.780 & 3.756 & 4.731 & 7.763 & 10.006 \\
$<\lambda>$ rolling & 0.0006 & 0.0038 & 0.0018 & 0.0160 & 0.0849 & 0.1123 \\
$\mathrm{~V}_{\mathrm{v}}$ cube & 0.0007 & 0.0009 & 0.0015 & 0.0060 & 0.0065 & 0.0006 \\
$\mathrm{~S}_{\mathrm{v}}$ cube & 1.875 & 4.770 & 4.556 & 5.812 & 14.575 & 17.497 \\
$<\lambda>$ cube & .0177 & 0.0544 & 0.1038 & 0.3042 & 0.7292 & 0.9583 \\
$\mathrm{~V}_{\mathrm{v}}$ total & .0188 & 0.0423 & 0.0490 & 0.1122 & 0.0729 & 0.0097 \\
$\mathrm{~S}_{\mathrm{v}}$ total & 2.425 & 3.626 & 4.475 & 5.841 & 10.772 & 11.641 \\
$<\lambda>$ total & & &
\end{tabular}

*The units of the $S_{\mathrm{v}}(\mathrm{i})$ are $\mu \mathrm{m}^{-1}$ and the units of the $\langle\lambda\rangle$ (i) are $\mu \mathrm{m}$. 
Table 2 Calculated Cahn-Hagel Velocity Parameters Using Eqn. (2).

\begin{tabular}{lcc}
\hline Texture component & $K_{c h}\left(\mu \mathrm{m} \cdot \mathrm{s}^{\alpha}{ }^{-1}\right)$ & $\alpha_{c h}$ \\
\hline random & $0.0534 \pm 0.0209$ & $0.41 \pm 0.06$ \\
rolling & $0.1442 \pm 0.0987$ & $0.57 \pm 0.10$ \\
cube & $0.1493 \pm 0.2560$ & $0.45 \pm 0.22$ \\
all & $0.0588 \pm 0.0253$ & $0.44 \pm 0.07$ \\
\hline
\end{tabular}

where $t$ is the annealing time, by employing the calculational method of Vandermeer and Juul Jensen (1994b). The interface-averaged velocities of each texture component were found to vary with time. The time dependence of each could be expressed by a formula of the type first given by English and Backofen (1964)

$$
\langle\mathrm{v}\rangle=\mathrm{K}_{\mathrm{ch}} \mathrm{t}^{-\alpha \mathrm{ch}}
$$

where the $\mathrm{K}_{\mathrm{ch}}$ and $\alpha_{\mathrm{ch}}$ were determined from least-squares calculations. The results of the calculations are listed in Table 2.

\section{Choice of Geometrical Model}

The findings from the Cahn-Hagel analysis of the growth velocities will be used as initial choices for the growth behavior in all subsequent geometrical modelling. The new recrystallized grains are assumed to grow as spheres. Thus, the radius function, $r_{i}$, for growth of a single grain of texture component $i$ may be obtained by integrating Eq. (2) to give

$$
\mathrm{r}_{\mathrm{i}}(\mathrm{t}-\tau)=\int_{\tau}^{\mathrm{t}}\langle\mathrm{v}\rangle_{\mathrm{i}} \mathrm{dt}=\frac{\mathrm{K}_{\mathrm{ch}}(\mathrm{i})}{1-\alpha_{\mathrm{ch}}(\mathrm{i})}(\mathrm{t}-\tau)^{1-\alpha_{\mathrm{ch}}(\mathrm{i})}
$$

where $\tau$ is the nucleation time. It is further assumed that $r_{i}(t=\tau) \approx 0$, i.e. at birth the nucleus size may be approximated as zero.

The other elements to consider for the model are the nucleation behavior (a constant nucleation rate versus a fixed number of pre-existing nuclei growing from zero time) and the impingement patterns (uniform impingement for randomly distributed nuclei versus anisotropically preferred impingement for clustered nuclei in linear or planar arrays (Vandermeer and Juul Jensen (1994a, 1995)). To decide upon and justify reasonable, experiment-rooted premises for these elements of the model, a preliminary analysis of the microstructural data was performed. This is accomplished by first examining the microstructural data in the context of a simple geometrical model for recrystallization (Vandermeer (1995), Vandermeer and Rath (1989a,b)) which ignores for the time being the individuality of the texture components, treating the whole as some average texture. The simple model assumes that the radius function is given by Eq. (3) and that nucleation is random (therefore impingements occur uniformly). In addition, the time dependence of the nucleation rate is assumed to be given by an equation of the form (Vandermeer (1995), Vandermeer, Masumura and Rath (1991)).

$$
\dot{\mathrm{N}}(\tau)=\mathrm{K}_{\mathrm{n}}(\mathrm{t}-\tau)^{\delta-1}
$$


where $\mathrm{K}_{\mathrm{n}}$ and $\delta$ are constants whose values are established by fitting, if possible, the experimental data to this simple model. It should be pointed out that $\delta$ is restricted by the model to have a value greater than zero.

For such a uniform impingement recrystallization model, it has been shown (Vandermeer, Masumura and Rath (1991)) that the time dependence and interdependence of the extended microstructural functions, $V_{v e x}$ and $S_{v e x}$, may be described analytically by the equations

$$
\begin{gathered}
\mathrm{V}_{\text {vex }}=-\ln \left(1-\mathrm{V}_{\mathrm{v}}\right)=\mathrm{Bt}^{\mathrm{k}} \\
\mathrm{S}_{\mathrm{vex}}=\frac{\mathrm{S}_{\mathrm{v}}}{1-\mathrm{V}_{\mathrm{v}}}=\mathrm{Kt}^{\mathrm{m}}
\end{gathered}
$$

and

$$
S_{\text {vex }}=\mathrm{CV}_{\mathrm{vex}}^{\mathrm{q}}
$$

where $\mathrm{k}, \mathrm{m}, \mathrm{q}, \mathrm{B}, \mathrm{K}$ and $\mathrm{C}$ are experimental constants that may be estimated by fitting the functions, Eqs. (5), (6) and (7) to the experimental data. The experimental constants may also be related to the model parameters, $\alpha_{c h}, \delta, K_{c h}$ and $K_{n}$ (Vandermeer, 1995). In Figures 1 and 2 the experimental data are plotted in a log-log format so as to evaluate the constants in Eqs. (5), (6) and (7). The relationships are described reasonably well by straight lines. For our purposes, the most instructive of the experimental parameters are $\mathrm{k}, \mathrm{m}$ and $\mathrm{q}$, the slopes of the lines shown in Figures 1 and 2, whose values, $1.20 \pm .05,0.72 \pm .03$ and $0.63 \pm .02$, respectively, were determined by a least-squares calculation.

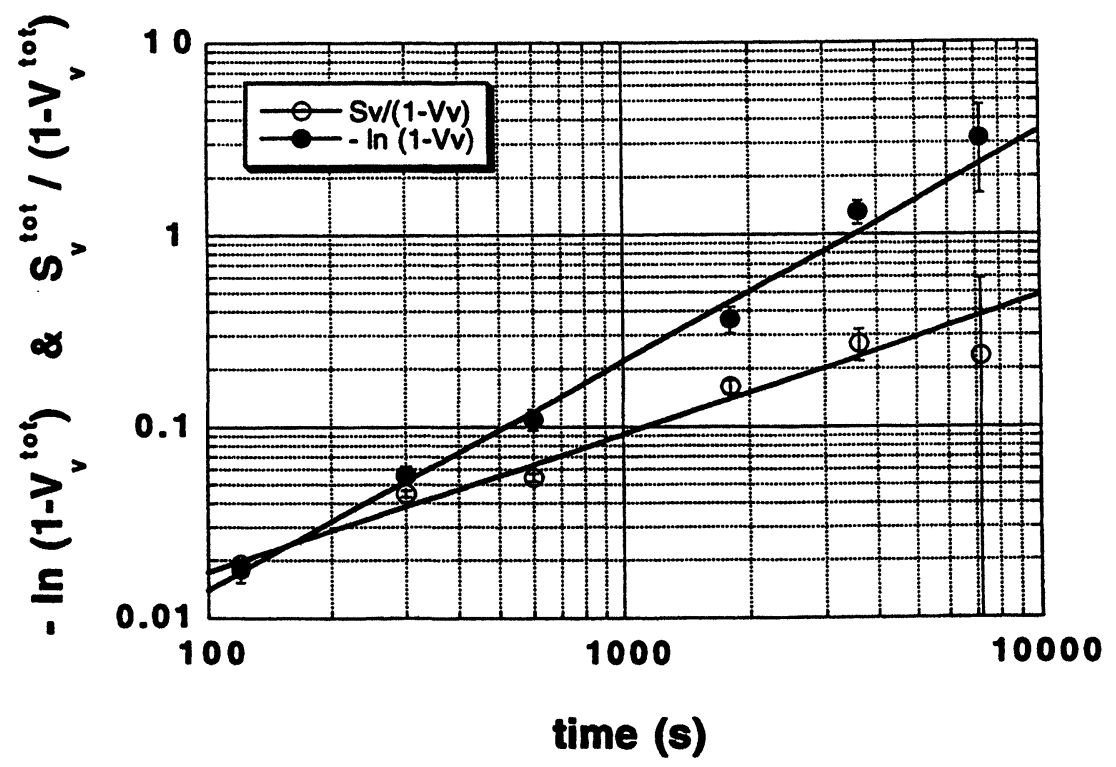

Figure 1 Log-log plots of $-\ln \left(1-V_{v}\right)$ and $S_{v} /\left(1-V_{v}\right)$ versus annealing time for the overall recrystallization of cold-rolled AA 1050 aluminum. Curves are based on a random nucleation model. 


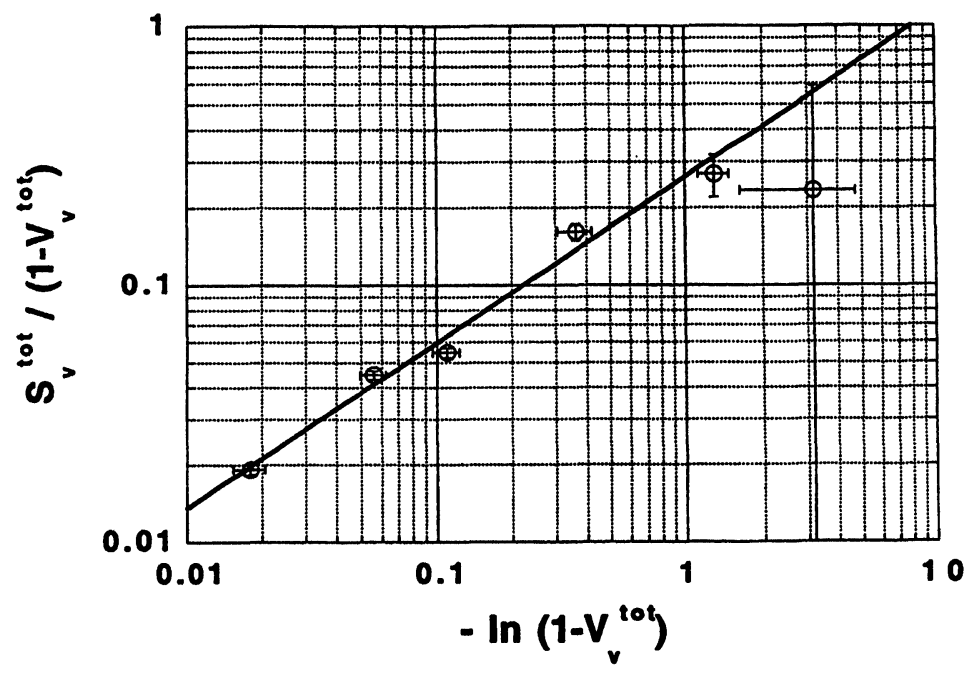

Figure 2 Log-log plots of $S_{v} /\left(1-V_{v}\right)$ versus $-\ln \left(1-V_{v}\right)$ illustrating the microstructural path for the overall recrystallization of cold-rolled AA 1050 aluminum. Curve is based on a random nucleation model.

Superficially, it would seem from the way the data agrees (Figures 1 and 2) with Eqs. (5), (6) and (7), that a simple, uniform impingement recrystallization model should describe the overall recrystallization of this aluminum adequately. This is found not to be the case when the model parameters are determined from $\mathrm{k}$ and $\mathrm{m}$. For the model, $\alpha_{\mathrm{ch}}=1-\mathrm{k}+\mathrm{m}$ and $\delta=3 \mathrm{~m}-2 \mathrm{k}$ (Vandermeer, 1995). Using the experimental values indicated above, it was calculated that $\alpha_{\mathrm{ch}}=0.52 \pm .06$ and $\delta=-0.24 \pm .13$. While $\alpha_{\text {ch }}$ appears reasonable (compared $0.52 \pm .06$ with $0.44 \pm .07$ in Table 2), negative values of $\delta$ are forbidden by the simple, uniform impingement model (Vandermeer, Masumura and Rath, 1991 and Vandermeer, 1995). That observation plus the result that $\mathrm{q}(=\mathrm{m} / \mathrm{k})$ is less than its minimum possible value of $0.67(\mathrm{q}=0.63 \pm .02$ and $\mathrm{m} / \mathrm{k}=0.60 \pm .04$ ) for randomly nucleated, nucleation and growth processes argues that the recrystallization nuclei are at least partly clustered and are not distributed in a way that is sufficiently random. Computer simulation also verified that a random nucleation recrystallization process would not be acceptable. As Figure 3 shows, the size distribution computed from a simulation of a randomly nucleated recrystallization process (solid line) is much too broad compared to the experimental distribution (the histogram itself). Too many larger grains are predicted by the simulation. In addition a randomly nucleated simulation revealed a much too strong a time dependence for $V_{v}$ and $S_{v}$ compared to experiment. So, to assume that nucleation is random is inappropriate. This same conclusion was also deduced for the aluminum studied in the earlier investigation (Vandermeer and Juul Jensen, 1994a).

To this point the analysis has demonstrated that a geometric model for recrystallization must deviate somewhat from a random nucleation outcome and that a reasonable model must consider some degree of nuclei clustering. Two such idealized, geometrical clustering arrangements can be conceived for which analytical formalisms may be derived (Vandermeer, 1995 and Vandermeer and Masumura, 1992). In the first, linear arrays or colonies of closely spaced grain nuclei may be envisioned such that because 


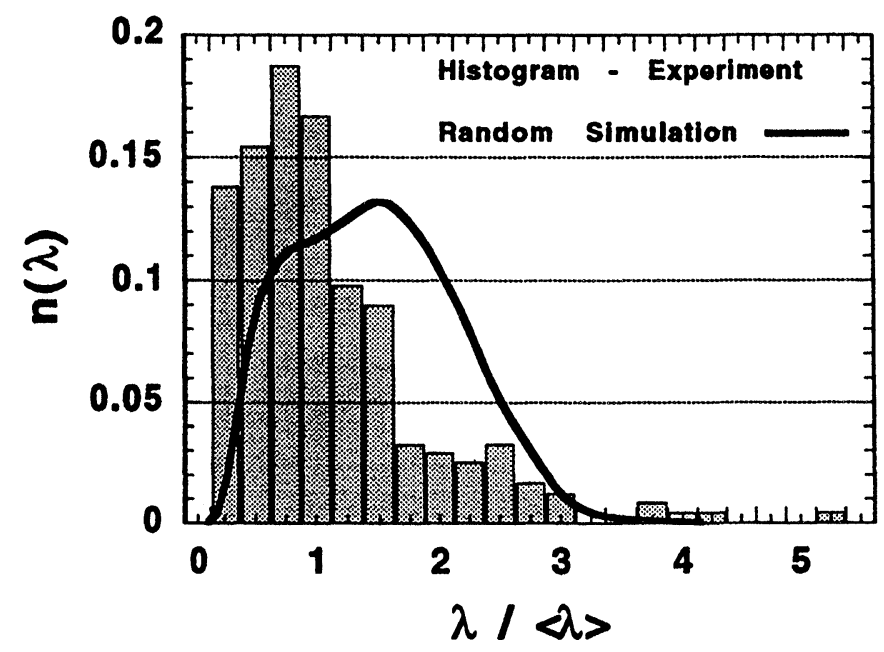

Figure 3 Plot of frequency versus normalized grain size (linear intercept values) for a fully recrystallized AA 1050 aluminum. The histogram represents the experimental EBSP data and the solid curve represents simulated results based on a random nucleation model.

of their closeness during growth, preferred, or anisotropic impingement occurs rapidly, blocking the new grain's growth in one dimension. This behavior is superimposed upon a uniform impingement in the grain's other growth dimensions. Such a model was quite effective for describing recrystallization of aluminum deformed $40 \%$ by rolling where the principle sites for nucleation were the deformed grain edges (triple junctions) (Vandermeer and Gordon, 1959 and Vandermeer and Masumura, 1992). This type of model also seemed appropriate for describing recrystallization in a more heavily rolled commercial aluminum containing particles (Vandermeer and Juul Jensen, 1994a), although in this case the linear colonies of new grains could not unequivocally be associated with the deformed grain edges.

To illustrate what effect preferential anisotropic (linear) impingement has on recrystallization kinetics, master curves depicting the time dependence of the functions, $V_{v}$ and $S_{v}$ are shown in Figure 4. These curves are for a special case of linearly clustered nucleation of pre-existing nuclei, no time dependent nucleation and a single texture component system. Note that $\mathrm{N}_{\mathrm{o}}$ and $\mathrm{L}$ are model parameters related to nucleation and that time, $t$, is embedded in the parameter, $K_{l u}$, which is given by

$$
\mathrm{K}_{\mathrm{lu}}=2 \mathrm{~N}_{\mathrm{o}} \frac{\mathrm{K}_{\mathrm{ch}}}{1-\alpha_{\mathrm{ch}}} \mathrm{t}^{1-\alpha_{\mathrm{ch}}}
$$

The $\mathrm{K}_{\mathrm{ch}}$ and $\alpha_{\mathrm{ch}}$ are the growth parameters which may be determined from a CahnHagel growth rate analysis as described earlier. The interpretation of the curves in Figure 4 is as follows: At very small $K_{l u}$ (time), the new recrystallized grains are growing essentially freely in all three growth dimensions and any impingement occurs at random. On the other hand, at very large values of $\mathrm{K}_{\mathrm{lu}}$ (time), the growth in one dimension of each grain is completely "pinched off" due to preferential impingement, the recrystallized grains are growing essentially two dimensionally as thickening 


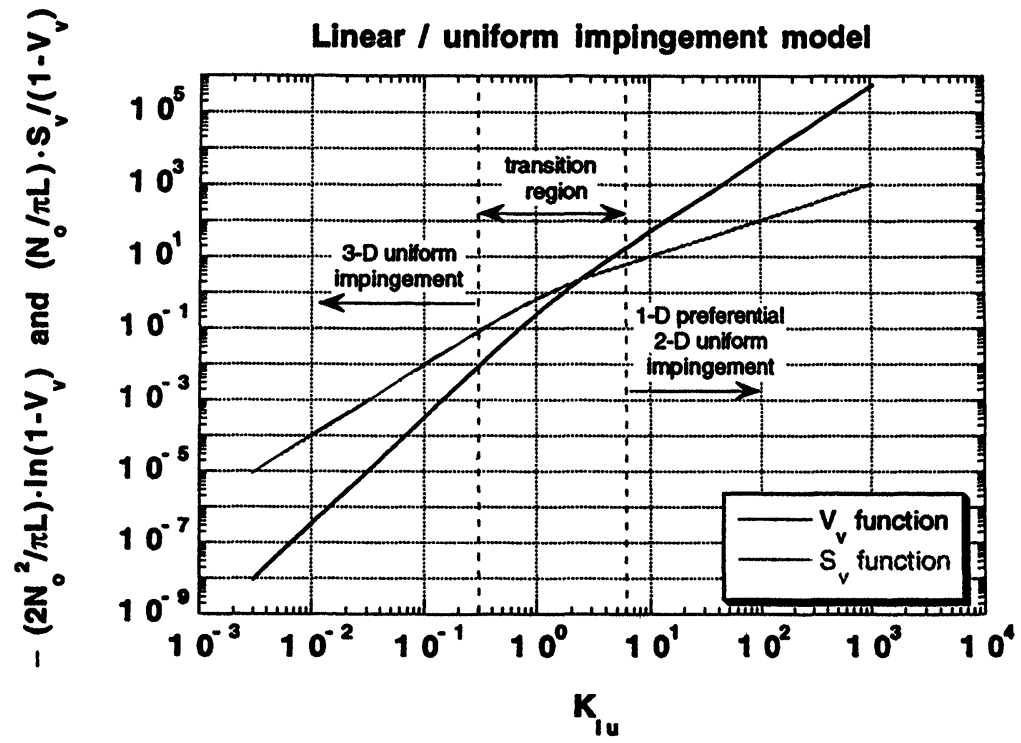

Figure 4 Normalized model kinetic functions for $V_{v}$ and $S_{v}$ plotted versus $K_{l u}$ (time) for a special case with clustered nucleation of preexisting nuclei, linear impingements and a single texture component.

"strings of beads" and in those two freely growing dimensions impingement takes place uniformly.

The transition region, i.e. intermediate times, represents the situation where preferential (or anisotropic) impingement in one growth direction begins to manifest itself more and more frequently with time and so becomes increasingly pervasive.

The second possible type of geometrically-clustered arrangement can be imagined as idealized planar arrays of close grain nuclei. Again, because the grains are near to one another during growth, preferred impingement causing growth to cease in two dimensions, is superimposed upon uniform impingements in the third growth dimension. Nucleation of recrystallized grains in deformation bands or at the deformed grain boundaries would exemplify cases where this kind of representation might be anticipated. A recent quantitative description of recrystallization in heavily rolled copper was based on just such a model (Vandermeer and Juul Jensen, 1995).

In both the linear and planar types of clustered arrangements, the recrystallization kinetics are marked by a transition from an initially faster rate, to a slower rate both because of the saturation of nucleation sites, if nucleation is active, and because of the change in the dimensionality of growth. The transition, however, is much more evident in the planar case than in the linear one where the changeover is more gradual (Vandermeer and Masumura, 1992).

Examining the value of $\mathrm{q}$ itself and the values of $\mathrm{k}$ and $\mathrm{m}$ relative to $3 \alpha_{\mathrm{ch}}$ and $2 \alpha_{\mathrm{ch}}$ and $2 \alpha_{\mathrm{ch}}$ and $\alpha_{\mathrm{ch}}$, respectively (these values refer to the slopes of the initial and final portions of the $V_{v}$ and $S_{v}$ curves shown in Figure 4), allows an assessment to be made of the experimental data within the context of a clustered nucleation model. Taken altogether the relationships suggest that a linear, preferred impingement clustering model would fill the requirements for the impingement pattern in this aluminum. 
Furthermore, from the experimental values of $\mathrm{q}, \mathrm{k}$ and $\mathrm{m}$, it seems reasonable to expect the recrystallization behavior to fall in the transition region of the model in the early stages of recrystallization with fully "pinched off" impingement occurring during the later stages of recrystallization. Why the linear impingement model was selected over the planer model is due to the fact that the experimental data appear to show only modest evidence of the expected transition whereas a much more noticeable transition would be expected if a planar impingement model had been supposed. From the lack of a more dominant transition, it may also be inferred that there is little or no time dependent nucleation. Indeed this was the type of model that was used to quantify nucleation and growth in the previously studied aluminum (Vandermeer and Juul Jensen, 1994a).

Based on the preceding arguments, the nucleation and growth model selected to represent recrystallization of the aluminum studied in this investigation has the following characteristics: 1) There are three texture components, random, rolling and cube. 2) The recrystallization nuclei are clustered in colonies which may be idealized for mathematical purposes as linear arrays of nuclei. 3) Mathematically, the nuclei are assumed to be randomly placed along the array length with regard to both position and texture component type. 4) All the recrystallization nuclei pre-exist and begin growing at annealing time, $t=0.5$ ) The shape of each growing grain while unimpinged is spherical. 6) The growth of each grain may be described by the radius function given by Eq. (3).

The mathematical formulation of the microstructural properties, $V_{v}(i)$ and $S_{v}(i)$ based on these premises will not be detailed here as it was presented already by Vandermeer and Juul Jensen (1994a). There, it was derived that the extended functions, $\mathrm{V}_{\text {vex }}(\mathrm{i})$ and $\mathrm{S}_{\mathrm{vex}}(\mathrm{i})$ are given analytically by the equations

$$
V_{\text {vex }}(i)=4 \pi L N_{o}(i) r_{i}{ }^{3} \int_{0}^{1} u\left\{\int_{0}^{\sqrt{1-u^{2}}} \exp \left(-2 r_{i} Z_{e}\right) d u\right\} d u
$$

and

$$
\mathrm{S}_{\mathrm{vex}}(\mathrm{i})=4 \pi \mathrm{L} \mathrm{N}_{\mathrm{o}}(\mathrm{i}) \mathrm{r}_{\mathrm{i}}^{2} \int_{0}^{1} \exp \left(-2 \mathrm{r}_{\mathrm{i}} \mathrm{Z}_{\mathrm{e}}\right) \mathrm{du}
$$

where

$$
Z_{e}=\sum_{j=1}^{3} N_{o}(j)\left(\left(\frac{r_{j}}{r_{i}}\right)^{2}+u-1\right)^{\frac{1}{2}}
$$

and $\mathrm{u}$ is the integration variable. The radius functions for each texture component are given by Eq. (3) and are expressed in terms of time (for this case $\tau$ is zero) and the parameters $\mathrm{K}_{\mathrm{ch}}(\mathrm{i})$ and $\alpha_{\mathrm{ch}}(\mathrm{i})$. The model parameter $\mathrm{L}$ in Eqs. (9) and (10) is the total array length per unit volume (Vandermeer and Juul Jensen, 1994a). The $\mathrm{N}_{0}(\mathrm{i})$ is the number of nuclei/grains per unit array length for component i. The product $\mathrm{N}_{\mathrm{o}}(\mathrm{i}) \mathrm{L}$ is the nuclei density, i.e. the number of nuclei per unit volume, for the texture component i. Equations (9) and (10) are related to the real space microstructural variables, $V_{v}(i)$ and $S_{v}(i)$ by the formulae

$$
\frac{\mathrm{dV}_{\mathrm{v}}(\mathrm{i})}{\mathrm{dV}_{\mathrm{vex}}(\mathrm{i})}=1-\mathrm{V}_{\mathrm{v}}(\text { total })
$$


and

$$
S_{\text {vex }}(i)=\frac{S_{v}(i)}{1-V_{v}(\text { total })}
$$

For calculational purposes Eq. (11) may be expressed in integrated form as

$$
\mathrm{V}_{\text {vex }}(\mathrm{i})=\int_{0}^{\mathrm{w}} \mathrm{R}(\mathrm{w}) \mathrm{dw}
$$

where $\mathrm{w}=-\ln \left[1-\mathrm{V}_{\mathrm{v}}(\mathrm{i})\right.$ and $\mathrm{R}(\mathrm{w})=\left[1-\mathrm{V}_{\mathrm{v}}(\mathrm{i})\right] /\left[1-\mathrm{V}_{\mathrm{v}}(\right.$ total $\left.)\right]$.

\section{Application of the Linear Impingement Model to Experiment}

For each texture component, the microstructural properties, $V_{\text {vex }}(i, \exp )$ and $S_{\text {vex }}(i, \exp )$, at each experimental annealing time were estimated using the data in Table 1 either directly with Eq. (12), as in the case of $S_{\text {vex }}(i, \exp )$ or indirectly, as for $V_{\text {vex }}(i, \exp )$ by integration using a polynomial function for $\mathrm{R}$ (wi) to represent the data and Eq. (13). Next, the model functions, $V_{\text {vex }}(\mathrm{i}, \mathrm{mod})(\mathrm{Eq} .9)$ and $\mathrm{S}_{\text {vex }}(\mathrm{i}, \mathrm{mod})(\mathrm{Eq} 10)$, were calculated numerically by computer after initial guesses for the model parameters (the $\mathrm{N}_{\mathrm{o}}(\mathrm{i})$ 's and the $\mathrm{L}$ ) are made. The initial values of the $\mathrm{K}_{\mathrm{ch}}(\mathrm{i})$ 's and $\alpha_{\mathrm{ch}}(\mathrm{i})$ 's were taken to be the previously determined values listed in Table 2 . The data and the function values so determined were plotted together on the same graph and the quality of the fit between the two was judged visually. If the fit was poor, the model parameters were adjusted, the model functions recalculated and a new plot constructed and evaluated. This trial and error procedure was repeated as many times as was necessary until the model functions emulated all the data well. The model parameters that accomplished this fit, then may be regarded as the "best guess" model parameters. The model parameters that were deduced are tabulated in Table 3. The quality of the experimental fit is illustrated in Figures 5 and 6. Eqs. (11) and (12) were employed to calculate the model functions, $V_{v}(i, m o d)$ and $S_{v}(i, m o d)$ plotted in these figures from the $V_{v e x}(i, m o d)$ and $S_{\text {vex }}(i, m o d)$ functions that were used to fit the data and to determine the model parameters. The $S_{v}(i, m o d)$ could be computed directly from Eq. (12) while an integration, analogous to that noted above, was necessary to estimate the $V_{v}(i, m o d)$. In these calculations, the total volume fraction recrystallized was estimated from the model functions by the formula

$$
\mathrm{V}_{\mathrm{v}}(\text { total })=1-\exp -\left(\sum_{\mathrm{i}=1}^{3} \mathrm{~V}_{\mathrm{vex}}(\mathrm{i})\right)
$$

From the model functions for the $\mathrm{V}_{\mathrm{v}}(\mathrm{i})$ 's and the $\mathrm{N}_{\mathrm{o}}(\mathrm{i})$ model parameters, a projection of the texture volume fraction at the end of recrystallization and a prediction of the

Table 3 Recrystallization Model Parameters for AA 1050 Aluminum.

\begin{tabular}{llll}
\hline Texture component & Random & Rolling & Cube \\
\hline$\alpha_{\mathrm{ch}}$ & 0.49 & 0.49 & 0.45 \\
$\mathrm{~K}_{\mathrm{ch}} \mu \mathrm{m}_{\mathrm{m}} \cdot \mathrm{s}_{\mathrm{ch}}-1$ & 0.0847 & 0.0836 & 0.099 \\
$\mathrm{~N}_{\mathrm{o}} \mu \mathrm{m}^{-1}$ & 0.07 & 0.034 & 0.002 \\
$\mathrm{~L} \mu \mathrm{m}^{-2}$ & & 0.00398 & \\
\hline
\end{tabular}




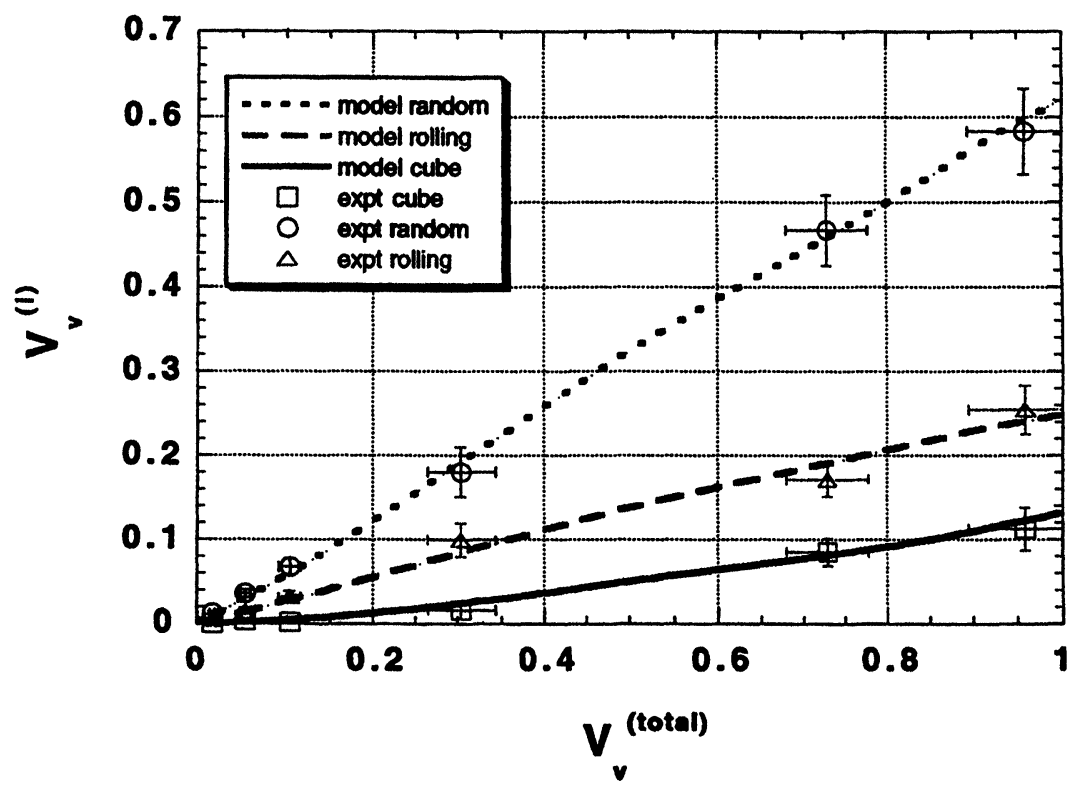

Figure 5 Plot of $\mathrm{V}_{\mathrm{v}}(\mathrm{i})$ versus $\mathrm{V}_{\mathrm{v}}$ (tot) comparing the "best guess" linear impingement model (curves) with experiment (data points) for recrystallization of the cube, rolling and random texture components of AA 1050 aluminum.

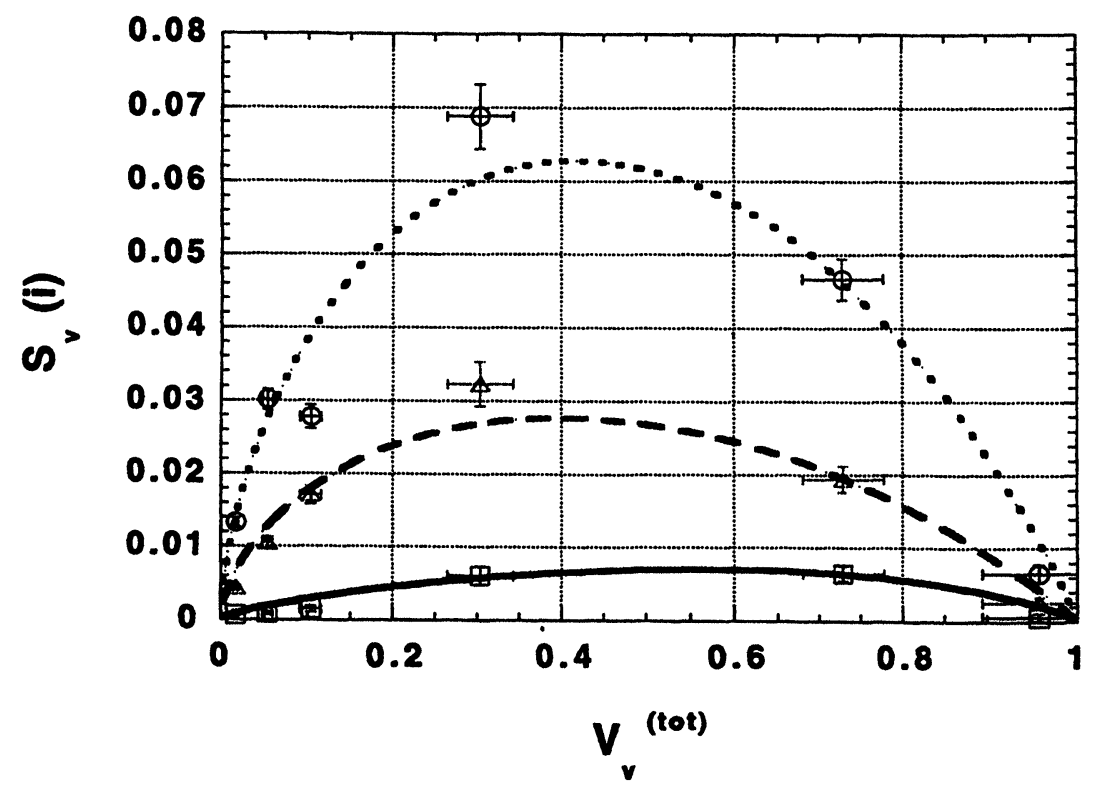

Figure 6 Plot of $S_{v}(i)$ versus $V_{v}(t o t)$ comparing the "best guess" linear impingement model (curves) with experiment (data points) for recrystallization of the cube, rolling and random texture components of AA 1050 aluminum. 
Table 4 As-Recrystallized Grain Sizes for AA1050 Aluminum.

\begin{tabular}{lcccccc}
\hline $\begin{array}{l}\text { texture } \\
\text { component }\end{array}$ & $\begin{array}{c}V_{v}(\text { model }) \\
\text { (from Fig.3) }\end{array}$ & $\begin{array}{c}V_{v} \\
\text { simulation }\end{array}$ & $\begin{array}{c}N_{v}(\text { model }) \\
\mathrm{mm}^{-3}\end{array}$ & $\begin{array}{c}\langle\lambda>\text { model } \\
\mu m\end{array}$ & $\begin{array}{c}<\lambda>\text { simul } \\
\mu m\end{array}$ & $\begin{array}{c}<\lambda>\text { expmt } \\
\mu m\end{array}$ \\
\hline random & 0.62 & 0.60 & 279000 & 9.8 & 10.9 & $11.3 \pm 5.7$ \\
rolling & 0.25 & 0.26 & 135000 & 9.2 & 10.6 & $10.0 \pm 4.7$ \\
cube & 0.13 & 0.14 & 8000 & 19 & 18.3 & $17.5 \pm 8.9$ \\
\hline
\end{tabular}

mean fully recrystallized grain size of each texture component can be realized. The texture fraction at the end of recrystallization can be estimated by extrapolation of the $\mathrm{V}_{\mathrm{v}}(\mathrm{i})$ functions to $\mathrm{V}_{\mathrm{v}}($ total $)=1$ in Figure 5. The mean recrystallized grain sizes can be deduced from stereological arguments put forth by Underwood (1968). In a spacefilled assembly of fully-recrystallized grains, it can be shown that the relationship between the number of grains per unit volume, $N_{v}$, and the average linear-intercept grain size, $\langle\lambda\rangle$, is given by (see Table $4-2$ on p 94 of Underwood (1968))

$$
\langle\lambda\rangle=\mathrm{F} \cdot\left(\frac{1}{N_{\mathrm{v}}}\right)^{\frac{1}{3}}
$$

where $\mathrm{F}$ is a constant having a value between 0.687 and 0.778 depending on the model of the grain structure that is selected. For our approximate purposes the value of 0.75 proposed by Kaiser (Underwood, 1968) will be assumed for $F$ with no further justification. To express Eq. (15) in terms of the individual texture components, it must be modified to

$$
\langle\lambda\rangle(i)=\frac{3}{4} \cdot\left(\frac{V_{v}(i)}{N_{v}(i)}\right)^{\frac{1}{3}}
$$

where $\mathrm{i}$ refers to a specific texture component and $\mathrm{V}_{\mathrm{v}}(\mathrm{i})$ is the model-extrapolated volume fraction of component $i$ at the completion of recrystallization. The $N_{v}(i)$ 's are determined from the product of the model constants, $N_{0}(i)$.L. So the model through Eq. (16) can be used to predict what the as-recrystallized, linear-intercept grain sizes should be for each texture component. Table 4 compares the as-recrystallized grain sizes calculated from the model with those experimentally measured for AA1050 aluminum. The model predictions are well within the scatter of the experimental values.

\section{Computer Simulation of Analytical Model}

The computer simulation creates a "specimen" which can be interrogated in much the same way a metallographic specimen is sectioned and analyzed by stereological methods. Therefore, from the simulation a recrystallized grain size distribution may also be generated which can be compared with experiment. Furthermore, geometrical modeling by computer simulation allows some of the ideality of the mathematical description to be relaxed. It provides more flexibility as to how the nucleation sites are distributed. Thus, for example, instead of the long straight lines that the mathematical approach requires of the linear impingement model (Vandermeer and Juul Jensen, 1994a), a series of segmented lines can be generated in the computer simulation (Juul Jensen and Vandermeer, 1995). Also, computer simulations allow slight adjustments to be made in the model parameters if necessary. 
The segmented line model for nucleation site locations proved to be an extremely good one in terms of its ability to match all the earlier experimental data on aluminum including the individual texture component data (Juul Jensen and Vandermeer, 1995) Using as initial input the model parameters deduced from the analytical modeling approach (Table 3), a number of computer simulations were run using the segmented line arrangement. Thus, a string of ten lines of randomly chosen directions were connected end to end to create a longer segmented (jagged) line. The number of nuclei placed on each line was varied from simulation to simulation, from one (similar to a random nucleation model) up to an average of ten for a total linear density in each case of $0.106 \mathrm{~mm}^{-1}$, i.e. $\Sigma \mathrm{N}_{0}(\mathrm{i})$ in Table 3 . Each simulation was run until recrystallization was complete. The simulation with the more closely spaced nuclei (10 per line segment) gave the best agreement with experiment. In Table 4 are listed the texture fractions and as-recrystallized, linear intercept grain sizes based on this simulation. These compare favorably with the experimental values and the analytical model calculations. In Figures $7 \mathrm{a}-\mathrm{c}$ the simulated grain size distributions (solid lines) for the model are superimposed upon the experimental histograms. The agreement is considered reasonable.

\section{DISCUSSION}

One problem with matching experimental data to an analytical model is the matter of uniqueness. It can not be proven that there is no other nucleation and growth microstructural representation that can describe the data equally as well as the one selected. Thus, the analytical model for AA1050 aluminum represented by the model parameters listed in Table 3 may not be unique. Nevertheless, it must be regarded as a "best guess" by virtue of the fact that all the measured microstructural characteristics are in agreement. The computer simulation results add further credence to the "best guess" model as Table 4 and Figures 7 a $-c$ reveal.

The combined stereological, analytical and computer simulation approach to geometrical modeling as detailed above is capable of describing many aspects of microstructural evolution during recrystallization of aluminum. When comparisons are made between the nucleation and growth behavior of the present aluminum and the earlier aluminum, there are many similarities but also a few differences. Certainly the essence of the microstructural model that describes recrystallization in the two materials is the same. Only in the details, i.e. the model nucleation and growth parameters, are the distinctions evident. Much of the kinetic difference can be ascribed to the disparity in the temperature of annealing which was $280^{\circ} \mathrm{C}$ for the current material and $253^{\circ} \mathrm{C}$ for the earlier material. Thus, the time-dependencies of $V_{v}$ and $S_{v}$ for the two materials can be rationalized if the activation energy for growth were approximately $171 \mathrm{kjoule} / \mathrm{mole}$. In the earlier study of aluminum (Juul Jensen and Vandermeer, 1995) the model, unlike in the present case, was unable to predict the as-recrystallized grain sizes correctly and the experimental grain sizes were about twice as large as the model projections. It would seem then that the analysis gave model parameters which were not the best ones in the earlier case. In the current study of a similar aluminum, that discrepancy does not exist even though the main features of the model are similar. The new analysis obviously allowed improved model parameters to be determined. The difference may be the result of 1) processing the aluminum to yield a more homogeneous pre-deformation precipitate state and 2) improvements in the EBSP measurements of the stereological properties themselves. 


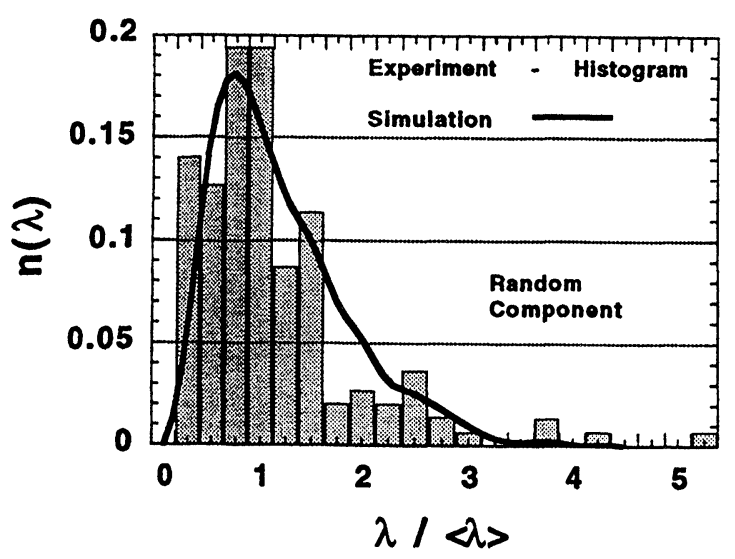

(a)

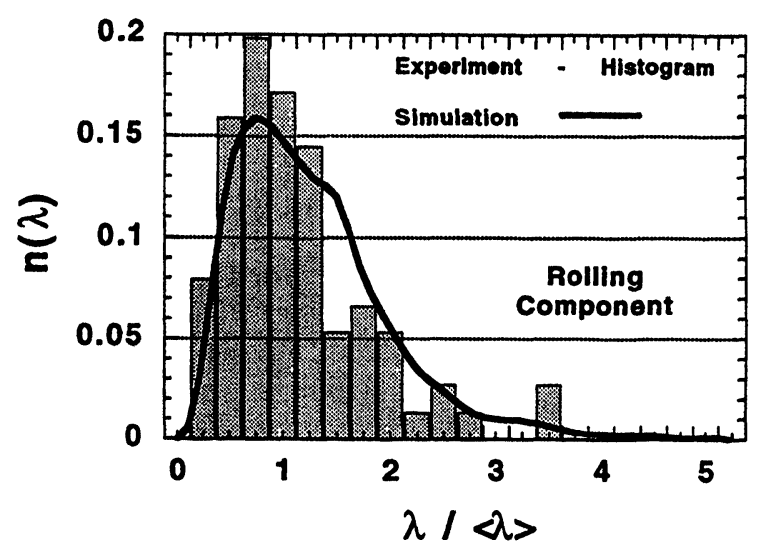

(b)

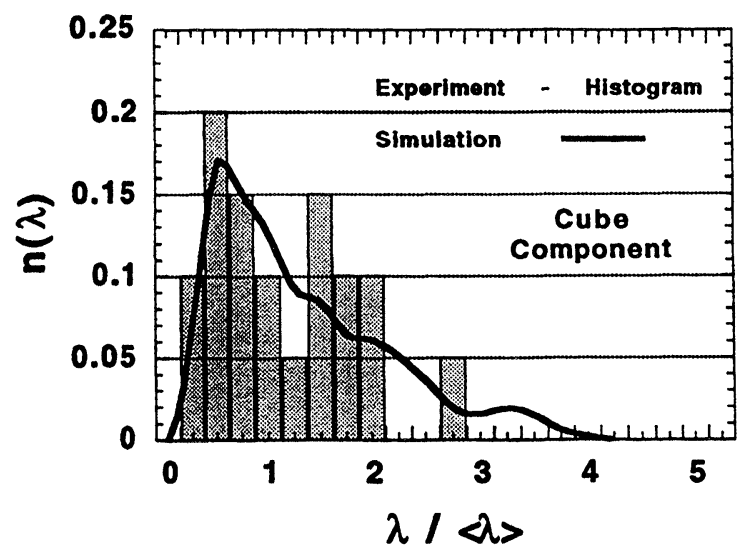

(c)

Figure 7 Plot of frequency versus normalized grain size (linear intercept values) for a fully recrystallized AA 1050 aluminum. The histograms represent the experimental EBSP data and the solid curves represent simulated results based on the clustered, linear impingement nucleation model. (a) random texture component, (b) rolling texture component and (c) cube texture component. 


\section{SUMMARY}

The microstructural evolution during isothermal recrystallization of AA 1050 aluminum cold-rolled $90 \%$ was analysed quantitatively by means of stereology, analytical modelling and computer simulation. A complete nucleation and growth model was deduced which described in detail both microstructural and textural development of the individual texture components including the volume fraction recrystallized, the interfacial area density separating recrystallized grains from the deformed regions, the texture fraction and the as-recrystallized grain sizes and grain size distributions.

\section{Acknowledgements}

This paper is dedicated to the memory of Professor William Hsun $\mathrm{Hu}$ who died recently. The authors wish to acknowledge the many significant contributions Professor $\mathrm{Hu}$ made to the subjects of recrystallization, grain growth and textures of metallic materials. His friendship and collegiality will be sorely missed. Also, appreciation is expressed for the support of the Naval Research Laboratory under the sponsorship of the Office of Naval Research of the U.S. Department of Navy.

\section{References}

Beck, P. and Hu, H. (1966). The origin of recrystallization textures, in Recovery, Recrystallization and Textures, ed. H. Margolin, ASM, Metals Park Ohio, pp. 393-433.

Beers, Y. (1957). Introduction to the Theory of Error, Addison-Wesley Publ. Co., Reading, Mass.

Cahn, J. W. and Hagel, W.C. (1960). Theory of the pearlite reaction, in Decomposition of Austenite by Diffusional Processes, eds. Z.D. Zackey and H.I. Aaronson, Interscience Publ., N.Y., pp. $131-196$

Cahn, J.W. and Hagel, W.C. (1963). Divergent pearlite in a manganese eutectoid steel, Acta Metallurgica, 11, pp. 561-574.

English, A.T. and Backofen, W.A. (1964). Recrystallization in hot-worked silicon-iron, Transactions TMS-AIME, 230, pp. 396-407.

Hilliard, J.E. and Cahn, J.W. (1961). An evaluation of procedures in quantitative metallography for volume-fraction analysis, Transactions TMS-AIME, 221, pp. 344-352.

Hilliard, J.E. (1966). Applications of quantitative metallography in recrystallization studies, in Recovery, Recrystallization and Textures, ed. H. Margolin, ASM, Metals Park Ohio, pp. 267-286.

$\mathrm{Hu}, \mathrm{H}$. (1981). Recovery, recrystallization and grain growth, in Treatises in Metallurgy, eds. J.K. Tien and J.F. Elliott, TMS-AIME, Warrenton Ohio, pp 385-407.

Juul Jensen, D. (1992). Modelling of microstructure development during recrystallization, Scripta Metallurgica, 27, pp. 1551-1556.

Juul Jensen, D. and Vandermeer, R.A. (1995). Simulation of recrystallization microstructures and textures in multiple texture component materials. Proceedings 4th European Conference on Advanced Materials and Processes, EUROMAT'95, vol. F, pp. ?

Kohler, U. and Bunge, H.J. (1994). Calculation of the recrystallization textures of cubic metals, Material Science Forum, 157-162, pp. 1803-1808.

Mahin, K.W., Hanson, K. and Morris, J.W. Jr. (1980). Comparative analysis of the cellular and Johnson-Mehl microstructures through computer simulation. Acta Metallurgica, 28, pp. 443-453.

Tavernier, Ph. and Szpunar, J.A. (1991). Modelling of recrystallization textures, Acta Metallurgica et Materialia, 39, pp. 549-556; (1991). A Monte Carlo simulation applied to the modelling of nucleation of texture, ibid., 39, pp. 557-567.

Underwood, E.E. (1968). Surface area and length in volume, in Quantitative Microscopy, eds. R.T. DeHoff and F.N. Rhines, McGraw-Hill Book Co., N.Y., pp. 77-127.

Vandermeer, R.A. (1995). Analytical modeling of the kinetics of recrystallization, in Microstructural and Crystallographic Aspects of Recrystallization, eds. N. Hansen, D. Juul Jensen, Y.L. Liu and B. Ralph, Ris $\varnothing$ National Laboratory, Roskilde, Denmark, pp. 193-213.

Vandermeer, R.A. and Gordon, P. (1959). Edge-nucleated, growth-controlled recrystallization in aluminum., Transactions TMS-AIME, 215, pp. 577-588. 
Vandermeer, R.A. and Juul Jensen, D. (1994a). Modeling microstructural evolution of multiple texture components during recrystallization, Acta Metallurgica et Materialia, 42, pp. 2427-2436.

Vandermeer, R.A. and Juul Jensen, D. (1994b). On the estimation of Cahn-Hagel interface migration rates, Scripta Metallurgica et Materialia, 30, pp. 1575-1580.

Vandermeer, R.A. and Juul Jensen, D. (1995). Quantifying recrystallization nucleation and growth kinetics of cold-worked copper by microstructural analysis, Metallurgical and Materials Transactions, $A, 26$ A, pp. 2227-2235.

Vandermeer, R.A., Masumura, R.A. and Rath, B.B. (1991). Microstructural paths of shape-preserved nucleation and growth transformations. Acta Metallurgica et Materialia, 39, pp. 383-389.

Vandermeer, R.A. and Masumura, R.A. (1992). The microstructural path of grain boundary nucleated phase transformations. Acta Metallurgica et Materialia, 40, pp. 877-886.

Vandermeer, R.A. and Rath, B.B. (1989a). Modeling recrystallization kinetics in a deformed iron single crystal, Metallurgical Transactions A, 20A, pp 391-401.

Vandermeer, R.A. and Rath, B.B. (1989b). Modeling of recrystallization, in Materials Architecture, eds. J.B. Bilde-Sørensen, N. Hansen, D. Juul Jensen, T. Leffers, H. Lilholt and O.B. Pedersen, Ris $\varnothing$ National Laboratory, Roskilde, Denmark, pp 589-599.

Woldt, E. and Juul Jensen, D. (1995). Recrystallization kinetics in copper: comparison between techniques, Metallurgical and Materials Transactions A, 26A, pp 1717-1724. 